\title{
A MAXIMAL DESCRIPTION FOR THE REAL INTERPOLATION METHOD IN THE QUASI-BANACH CASE.
}

\author{
JÖRAN BERGH and FERNANDO COBOS ${ }^{1}$
}

\section{Abstract}

We give a maximal description in the sense of Aronszajn-Gagliardo for the real method in the category of quasi-Banach spaces.

Let $\bar{A}=\left(A_{0}, A_{1}\right)$ be a couple of quasi-Banach spaces, $0<q \leq \infty$ and $0<\theta<1$. The real interpolation space $\bar{A}_{\theta, q}=\left(A_{0}, A_{1}\right)_{\theta, q}$ consists of all $a \in A_{0}+A_{1}$ which have a finite quasi-norm

$$
\begin{gathered}
\|a\|_{\theta, q}=\left(\sum_{m=-\infty}^{\infty}\left(2^{-\theta m} K\left(2^{m}, a\right)\right)^{q}\right)^{1 / q} \text { if } 0<q<\infty \\
\|a\|_{\theta, q}=\sup _{m \in Z}\left\{2^{-\theta m} K\left(2^{m}, a\right)\right\} \text { if } q=\infty
\end{gathered}
$$

where $K(t,$.$) is the K$-functional of Peetre, defined by

$$
K(t, a)=\inf \left\{\left\|a_{0}\right\|_{A_{0}}+t\left\|a_{1}\right\|_{A_{1}}: a=a_{0}+a_{1}, a_{i} \in A_{i}\right\} .
$$

As an example, let us recall that

$$
\left(L_{p_{0}}, L_{p_{1}}\right)_{\theta, q}=L_{q} \text { (equivalent quasi-norms) }
$$

provided $0<p_{0}, p_{1}, q \leq \infty, 0<\theta<1$ and $\frac{1}{q}=\frac{1-\theta}{p_{0}}+\frac{\theta}{p_{1}}$. We refer to the book by Bergh and Löfström [2] for full details on the real method.

The space $\left(A_{0}, A_{1}\right)_{\theta, q}$ is a quasi-Banach space, but if $\left(A_{0}, A_{1}\right)$ is a Banach couple and $1 \leq q \leq \infty$, then $\left(A_{0}, A_{1}\right)_{\theta, q}$ turns out to be a Banach space. Besides, working with Banach spaces, the real interpolation method can be described as a maximal functor in the sense of Aronszajn and Gagliardo [1]. Next we review that description.

\footnotetext{
1* Supported in part by DGICYT 9PB94-0252)

Received November 3, 1997.
} 
Given two Banach couples $\bar{A}=\left(A_{0}, A_{1}\right), \bar{B}=\left(B_{0}, B_{1}\right)$, we write $T \in \mathscr{L}(\bar{A}, \bar{B})$ to mean that $T$ is a linear operator from $A_{0}+A_{1}$ into $B_{0}+B_{1}$ whose restriction to each $A_{i}(\mathrm{i}=0,1)$ defines a bounded operator from $A_{i}$ into $B_{i}$. Set

$$
\|T\|_{\bar{A}, \bar{B}}=\max \left\{\|T\|_{A_{0}, B_{0}},\|T\|_{A_{1}, B_{1}}\right\}
$$

Operators ending in the couple $\bar{\ell}_{\infty}=\left(\ell_{\infty}, \ell_{\infty}\left(2^{-m}\right)\right)$ will be of special interest for us. Scalar sequence spaces are defined over $\mathbf{Z}$ and given any sequence of positive numbers $\left(w_{m}\right)$, we put

$$
\ell_{q}\left(w_{m}\right)=\left\{\left(\xi_{m}\right):\left\|\left(\xi_{m}\right)\right\|_{\ell_{q}\left(w_{m}\right)}=\left\|\left(w_{m} \xi_{m}\right)\right\|_{\ell_{q}}<\infty\right\} .
$$

Janson proved in [4] that the real method in the category of Banach spaces coincides with the maximal interpolation functor generated by the couple $\bar{\ell}_{\infty}$ and the intermediate space $\ell_{q}\left(2^{-\theta m}\right)$. In other words $\left(A_{0}, A_{1}\right)_{\theta, q}$ is equal to the space $H\left[\left(\ell_{\infty}, \ell_{\infty}\left(2^{-m}\right)\right) ; \ell_{q}\left(2^{-\theta m}\right)\right]\left(A_{0}, A_{1}\right)$ that consists of all elements $a \in A_{0}+A_{1}$ such that $T a \in \ell_{q}\left(2^{-\theta m}\right)$ for all $T \in \mathscr{L}\left(\bar{A}, \bar{\ell}_{\infty}\right)$. Moreover, the norm

$$
\|a\|_{H}=\sup \left\{\|T a\|_{\ell_{q}\left(2^{-\theta m}\right)}:\|T\|_{\bar{A}, \bar{\ell}_{\infty}} \leq 1\right\}
$$

is equal to $\|a\|_{\theta, q}$.

The proof is based on the Hahn-Banach theorem, so it does not work for quasi-Banach couples. In fact, as far as we are aware, no maximal description is known for the real method in the quasi-Banach case.

In a recent paper Cobos and Persson [3] pointed out that replacing $\bar{\ell}_{\infty}$ and $\ell_{q}\left(2^{-\theta m}\right)$ by other scalar sequence spaces is not a help in this problem. They also showed that a maximal description for each quasi-Banach couple $\left(A_{0}, A_{1}\right)$ can be given in terms of vector valued sequence spaces modelled on the sum $A_{0}+A_{1}$. But this is different from a description of the real interpolation method.

In this note we will establish a maximal description for the real method. The key of our approach is to deal with quasi-linear operators rather than linear operators. This is in harmony with the theory because the real method is inspired by the proof of the Marcinkiewicz interpolation theorem which works for quasi-linear operators.

Let $T$ be a mapping from a quasi-Banach space $A$ into a scalar sequence space $\omega$. We say that $T$ is quasi-linear with constant $C \geq 1$ if

$$
\begin{aligned}
|T(a+b)| & \leq C(|T a|+|T b|), a, b \in A \\
|T(\lambda a)| & =|\lambda||T a|, \lambda \in \mathrm{K}, \mathrm{a} \in A
\end{aligned}
$$

where the inequalities should be understood coordinatewise and $\mathrm{K}$ stands for the scalar field. 
Given any quasi-Banach couple $\bar{A}=\left(A_{0}, A_{1}\right)$ and $C \geq 1$, we denote by $\mathscr{2}_{C}\left(\bar{A}, \bar{\ell}_{\infty}\right)$ the collection of all those quasi-linear operators $T: A_{0}+A_{1} \rightarrow$ $\ell_{\infty}+\ell_{\infty}\left(2^{-m}\right)$ with constant $C$ whose restriction to $A_{i}(i=0,1)$ defines a bounded operator from $A_{i}$ into $\ell_{\infty}\left(2^{-i m}\right)$, i.e.

$$
\|T\|_{A_{i}, \ell_{\infty}\left(2^{-i m}\right)}=\sup \left\{\|T a\|_{\ell_{\infty}\left(2^{-i m}\right)}:\|a\|_{A_{i}} \leq 1\right\}<\infty .
$$

Set

$$
\|T\|_{\bar{A}, \bar{\ell}_{\infty}}=\max \left\{\|T\|_{A_{0}, \ell_{\infty}},\|T\|_{A_{1}, \ell_{\infty}\left(2^{-m}\right)}\right\} .
$$

Following the construction of Aronszajn and Gagliardo, given any quasiBanach couple $\bar{A}=\left(A_{0}, A_{1}\right), 0<\theta<1$ and $0<q<\infty$, we define

$$
\widetilde{H}_{C}\left[\left(\ell_{\infty}, \ell_{\infty}\left(2^{-m}\right)\right) ; \ell_{q}\left(2^{-\theta m}\right)\right]\left(A_{0}, A_{1}\right)=\widetilde{H}_{C ; \theta, q}\left(A_{0}, A_{1}\right)
$$

as the collection of all those $a \in A_{0}+A_{1}$ such that $T a \in \ell_{q}\left(2^{-\theta m}\right)$ for any $T \in \mathscr{Q}_{C}\left(\bar{A}, \bar{\ell}_{\infty}\right)$ and the quasi-norm

$$
\|a\|_{\widetilde{H}_{C}}=\sup \left\{\|T a\|_{\ell_{q}\left(2^{-\theta m}\right)}:\|T\|_{\bar{A}, \bar{\ell}_{\infty}} \leq 1\right\}
$$

is finite.

We next establish the result.

Theorem 1. Let $0<q \leq \infty, 0<\theta<1$ and let $\bar{A}=\left(A_{0}, A_{1}\right)$ be a quasi-Banach couple. Assume that the constant in the triangle inequality of $A_{i}$ is $C_{i}$ $(i=0,1)$ and put $C=\max \left\{C_{0}, C_{1}\right\}$. Then

$$
\left(A_{0}, A_{1}\right)_{\theta, q}=\widetilde{H}_{C}\left[\left(\ell_{\infty}, \ell_{\infty}\left(2^{-m}\right)\right) ; \ell_{q}\left(2^{-\theta m}\right)\right]\left(A_{0}, A_{1}\right)
$$

with

$$
\|a\|_{\theta, q} \leq\|a\|_{\widetilde{H}_{C}} \leq C\|a\|_{\theta, q} .
$$

Proof. Let $m \in \mathbf{Z}$ and $a, b \in A_{0}+A_{1}$. Given any decompositions $a=a_{0}+a_{1}, b=b_{0}+b_{1}$, with $a_{i}, b_{i} \in A_{i}$, it follows from

$$
\begin{aligned}
K\left(2^{m}, a+b\right) & \leq\left\|a_{0}+b_{0}\right\|_{A_{0}}+2^{m}\left\|a_{1}+b_{1}\right\|_{A_{1}} \\
& \leq C\left[\left(\left\|a_{0}\right\|_{A_{0}}+2^{m}\left\|a_{1}\right\|_{A_{1}}\right)+\left(\left\|b_{0}\right\|_{A_{0}}+2^{m}\left\|b_{1}\right\|_{A_{1}}\right)\right]
\end{aligned}
$$

that

$$
K\left(2^{m}, a+b\right) \leq C\left(K\left(2^{m}, a\right)+K\left(2^{m}, b\right)\right) .
$$

Hence the map $T: A_{0}+A_{1} \rightarrow \ell_{\infty}+\ell_{\infty}\left(2^{-m}\right)$ defined by $T a=\left(K\left(2^{m}, a\right)\right)$ belongs to $\mathscr{Q}_{C}\left(\bar{A}, \bar{\ell}_{\infty}\right)$. Moreover

$$
\|T a\|_{\ell_{\infty}\left(2^{-i m}\right)}=\sup _{m \in Z}\left\{2^{-i m} K\left(2^{m}, a\right)\right\} \leq\|a\|_{A_{i}}, a \in A_{i}, i=0,1
$$

so $\|T\|_{\bar{A}, \bar{\ell}_{\infty}} \leq 1$. 
Now take any $a \in \widetilde{H}_{C ; \theta, q}\left(A_{0}, A_{1}\right)$. Since

$$
\|a\|_{\theta, q}=\left\|\left(K\left(2^{m}, a\right)\right)\right\|_{\ell_{q}\left(2^{-\theta m}\right)}=\|T a\|_{\ell_{q}(2-\theta m)} \leq\|a\|_{\widetilde{H}_{C}}
$$

we derive that $a \in\left(A_{0}, A_{1}\right)_{\theta, q}$ and that the inclusion $\widetilde{H}_{C ; \theta, q}\left(A_{0}, A_{1}\right) \hookrightarrow\left(A_{0}, A_{1}\right)_{\theta, q}$ has quasi-norm $\leq 1$.

To check the converse inclusion we shall use an argument similar to the Banach case (see [4]). Given any $T \in \mathscr{Q}_{C}\left(\bar{A}, \bar{\ell}_{\infty}\right)$ with $\|T\|_{\bar{A}, \bar{\ell}_{\infty}} \leq 1$, we can represent $T a$ as $\left(T_{m} a\right)$ where $T_{m} \in \mathscr{Q}_{C}\left(\left(A_{0}, A_{1}\right),(\mathrm{K}, \mathrm{K})\right)$ with

$$
\left\|T_{m}\right\|_{A_{0}, \mathrm{~K}} \leq\|T\|_{A_{0}, \ell_{\infty}} \leq 1,
$$

and

$$
\left\|T_{m}\right\|_{A_{1}, 2^{-m} \mathrm{~K}} \leq\|T\|_{A_{1}, \ell_{\infty}\left(2^{-m}\right)} \leq 1
$$

If $a \in A_{0}+A_{1}$ and $a=a_{0}+a_{1}$ with $a_{i} \in A_{i}$, we get

$$
\left|T_{m} a\right| \leq C\left(\left|T_{m} a_{0}\right|+\left|T_{m} a_{1}\right|\right) \leq C\left(\left\|a_{0}\right\|_{A_{0}}+2^{m}\left\|a_{1}\right\|_{A_{1}}\right) .
$$

Whence

$$
\left|T_{m} a\right| \leq C K\left(2^{m}, a\right) .
$$

Let now $a \in\left(A_{0}, A_{1}\right)_{\theta, q}$. We obtain

$$
\begin{aligned}
\|T a\|_{\ell_{q}\left(2^{-\theta m}\right)} & =\left(\sum_{m=-\infty}^{\infty}\left(2^{-\theta m}\left|T_{m} a\right|\right)^{q}\right)^{1 / q} \\
& \leq C\left(\sum_{m=-\infty}^{\infty}\left(2^{-\theta m} K\left(2^{m}, a\right)\right)^{q}\right)^{1 / q}=C\|a\|_{\theta, q} .
\end{aligned}
$$

This shows the embedding $\left(A_{0}, A_{1}\right)_{\theta, q} \hookrightarrow \widetilde{H}_{C ; \theta, q}\left(A_{0}, A_{1}\right)$ and finishes the proof.

The real interpolation method can be equivalently defined by means of the J-functional

$$
J(t, a)=\max \left\{\|a\|_{A_{0}}, t\|a\|_{A_{1}}\right\}, a \in A_{0} \cap A_{1} .
$$

In that form it can be described as a minimal interpolation functor in the sense of Aronszajn and Gagliardo [1], as was proved by Janson [4] (for Banach spaces) and Mastylo [5] (for quasi-Banach spaces; see also [3]). This time the extension from the Banach case to the quasi-Banach case is easy because the minimal construction sets sequence spaces as domains of operators and because the proof in the Banach case does not require the HahnBanach theorem. Non-linear operators are not needed.

As a concluding remark, we would like to point out a connection between the main stability results of Cobos and Persson [3] for maximal and minimal methods and the classical stability (or reiteration) result for the real inter- 
polation method (see [2] and also [4]). In this situation, we restrict to the category of Banach spaces and bounded linear operators. Given any Banach couple $\left(A_{0}, A_{1}\right)$, and any numbers $0<\theta_{0}, \theta_{1}, \theta, \eta<1$ with $\theta=(1-\eta) \theta_{0}+\eta \theta_{1}$, we put

$$
F_{m}=\left(A_{0}+A_{1}, 2^{-\frac{\theta_{0} m}{\theta_{1}-\theta_{0}}} K\left(2^{\frac{m}{\theta_{1}-\theta_{0}}}, .\right)\right), G_{m}=\left(A_{0} \cap A_{1}, 2^{-\frac{\theta_{0} m}{\theta_{1}-\theta_{0}}} J\left(2^{\frac{m}{\theta_{1}-\theta_{0}}}, .\right)\right) .
$$

By [3], Thm. 1.4, we get

$$
\begin{aligned}
\left(\bar{A}_{\theta_{0}, \infty}, \bar{A}_{\theta_{1}, \infty}\right)_{\eta, q} & =H\left[\left(\ell_{\infty}, \ell_{\infty}\left(2^{-m}\right)\right) ; \ell_{q}\left(2^{-\eta m}\right)\right]\left(\bar{A}_{\theta_{0}, \infty}, \bar{A}_{\theta_{1}, \infty}\right) \\
& =H\left[\left(\ell_{\infty}\left(F_{m}\right), \ell_{\infty}\left(2^{-m} F_{m}\right)\right) ; \ell_{q}\left(2^{-\eta m} F_{m}\right)\right]\left(\bar{A}_{\theta_{0}, \infty}, \bar{A}_{\theta_{1}, \infty}\right) \\
& \hookrightarrow H\left[\left(\bar{A}_{\theta_{0}, \infty}, \bar{A}_{\theta_{1}, \infty}\right) ; \bar{A}_{\theta, q}\right]\left(\bar{A}_{\theta_{0}, \infty}, \bar{A}_{\theta_{1}, \infty}\right) \\
& \hookrightarrow \bar{A}_{\theta, q} .
\end{aligned}
$$

Similarly, using the minimal description of the real method and [3], Thm. 1.8 , we obtain

$$
\begin{aligned}
\bar{A}_{\theta, q} & \hookrightarrow G\left[\left(\bar{A}_{\theta_{0}, 1}, \bar{A}_{\theta_{1}, 1}\right) ; \bar{A}_{\theta, q}\right]\left(\bar{A}_{\theta_{0}, 1}, \bar{A}_{\theta_{1}, 1}\right) \\
& \hookrightarrow G\left[\left(\ell_{1}\left(G_{m}\right), \ell_{1}\left(2^{-m} G_{m}\right)\right) ; \ell_{q}\left(2^{-\eta m} G_{m}\right]\left(\bar{A}_{\theta_{0}, 1}, \bar{A}_{\theta_{1}, 1}\right)\right. \\
& =G\left[\left(\ell_{1}, \ell_{1}\left(2^{-m}\right)\right) ; \ell_{q}\left(2^{-\eta m}\right)\right]\left(\bar{A}_{\theta_{0}, 1}, \bar{A}_{\theta_{1}, 1}\right) \\
& =\left(\bar{A}_{\theta_{0}, 1}, \bar{A}_{\theta_{1}, 1}\right)_{\eta, q} .
\end{aligned}
$$

From these inclusions we get for $1 \leq q_{0}, q_{1}, q \leq \infty$

$$
\left(\bar{A}_{\theta_{0}, q_{0}}, \bar{A}_{\theta_{1}, q_{1}}\right)_{\eta, q}=\bar{A}_{\theta, q}
$$

i.e. the reiteration theorem.

\section{REFERENCES}

1. N. Aronszajn and E. Gagliardo, Interpolation spaces and interpolation methods, Ann. Mat. Pura Appl. 68 (1965), 51-118.

2. J. Bergh and J. Löstrom, 'Interpolation Spaces. An Introduction', Grundlehren Math. Wiss. 223, 1976.

3. F. Cobos, and L.-E. Persson, Real interpolation of compact operators between quasi-Banach spaces, Math. Scand. 82 (1998), 138-160.

4. S. Janson, Minimal and maximal method of interpolation, J. Funct. Anal. 44 (1981), 50-73.

5. M. Mastylo, On interpolation of some quasi-Banach spaces, J. Math. Anal. Appl. 147 (1990), 403-419.

DEPARTMENT OF MATHEMATICS CHALMERS UNIVERSITY OF TECNOLOGY AND UNIVERSITY OF GÖTEBORG S-41296 GÖTEBORG. SWEDEN

Email: bergh@math.chalmers.se
DEPARTAMENTO DE ANÁLISIS MATEMÁTICO FACULTAD DE MATEMÁTICAS UNIVERSIDAD COMPLUTENSE DE MADRID 28040 MADRID. SPAIN

Email: cobos@eucmax.sim.ucm.es 